\title{
„ICH HANTLE MICH OFT VON EINER INFORMATION ZUR NÄCHSTEN“. OPEN DATA - OPEN SCIENCE - OFFEN FÜR ALLE ODER GIBT ES DOCH NOCH BARRIEREN? EIN INTERVIEW MIT BARBARA LEVC
}

\author{
von Susanne Blumesberger und Barbara Levc
}

Zusammenfassung: Open Science und Open Data, sowie Fair-Data sind Schlagworte, die aus dem Wissenschaftsdiskurs kaum mehr wegzudenken sind. Wenig bis gar nicht beachtet wird jedoch, dass offene Daten oft nicht wirklich für alle potentiellen User offen sind. Personen mit unterschiedlichen Einschränkungen haben teilweise keinen Zugang zu Informationen. Diese Tatsache ist oft nicht bewusst. Viel zu selten werden betroffene Personen in die Überlegungen miteinbezogen. Frau Mag. ${ }^{a}$ Levc, selbst blind, am Zentrum Integriert Studieren ${ }^{1}$ an der Universität Graz tätig, hat freundlicherweise Auskunft über ihren Umgang mit Informationen gegeben.

Schlagwörter: Offene Daten; offene Wissenschaft; Barrierefreiheit

\section{„I OFTEN WORK FROM ONE PIECE OF INFORMATION TO THE NEXT“. OPEN DATA - OPEN SCIENCE - OPEN TO ALL OR ARE THERE STILL BARRIERS?}

\begin{abstract}
Open Science and Open Data as well as Fair-Data are buzzwords that have become an integral part of the academic discourse. However, little or no attention is paid to the fact that open data is often not really open to all potential users. Persons with different restrictions sometimes have no access to information. This fact is often unaware. Far too seldom, affected persons are included in the considerations. Ms. Levc, herself blind, working at the Center for Integrated Studying at the University of Graz, has kindly provided information about her handling of information.
\end{abstract}

Keywords: Open Data; Open Science; Accessibility

DOI: https://doi.org/10.31263/voebm.v72i2.2835

(c) Susanne Blumesberger, Barbara Levc

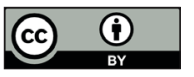

Dieses Werk ist lizenziert unter einer

Creative-Commons-Lizenz Namensnennung 4.0 International 
Es werden vermehrt offene Daten und eine möglichst transparente Wissenschaft gefordert. Damit soll der Zugriff auf wissenschaftlichen Output für alle gesichert werden. Immer mehr Universitäten verabschieden Forschungsdatenpolicies und geben damit Richtlinien vor, wie die Forschenden für ein sorgfältiges Datenmanagement zu sorgen haben. Ziel ist es, Daten nachhaltig zu sichern, mit möglichst freien Lizenzen zu versehen und so für alle nachnutzbar zu machen. ${ }^{2}$ „Für alle" schließt per se auch Menschen mit diversen Beeinträchtigungen (wie unterschiedlichen Sehbehinderungen, Blindheit, Gehörlosigkeit, Hörbeeinträchtigungen, motorischen oder kognitiven Beeinträchtigungen) ein. Selbst Menschen mit einer Farbfehlsichtigkeit, einer Störung, die recht weit verbreitet ist, fällt es jedoch oft schwer, sich aufWebsites oder in Publikationen zurechtzufinden, wenn wichtige Hinweise nur durch unterschiedliche Farben gekennzeichnet sind. Informationen nicht nutzen zu können, weil man aufgrund einer Behinderung keinen Zugang dazu hat bzw. selbst nichts beitragen, also publizieren zu können, bedeutet ausgegrenzt zu sein - im Alltag aber vor allem auch in der Wissenschaft.

Um diese Missstände zu beseitigen, wird einerseits technische Expertise benötigt, andererseits ist aber auch Expertlnnenwissen aus anderen Bereichen gefragt, denn eine perfekte Aufbereitung eines Dokuments muss nicht für alle gleichermaßen lesbar und verständlich sein. Eine enge Zusammenarbeit mit betroffenen Personen ist hier absolut notwendig, um nicht an den Bedürfnissen unterschiedlicher Anwenderlnnen vorbei zu arbeiten. Grundsätzliches Wissen, beispielsweise, wie man Texte barrierefrei gestaltet oder Bilder so beschreibt, dass sie auch nicht sehenden Personen zugänglich sind, kann recht rasch erworben werden. Es fehlt jedoch oft an der Umsetzung, da das jeweilige Problem von vielen Menschen ohne Einschränkungen gar nicht wahrgenommen wird. Jemand, der an keiner Farbsehstörung leidet, kann sich nur schwer vorstellen, dass für manche der rote und der grüne Button nicht oder kaum zu unterscheiden ist. Viele Mängel in einem System werden erst bewusst, wenn betroffene Nutzerlnnen nicht ohne fremde Hilfe auf Daten zugreifen können. An erster Stelle müsste deshalb Bewusstseinsbildung stehen, um problematische Situationen, mit denen viele Personen im Alltag konfrontiert sind, deutlich zu machen. Es wäre ebenso nötig, potentielle Hürden ausfindig zu machen, die den Weg zu offenen Daten versperren. Vor allem in Repositorien, aber auch in Katalogen wäre es deshalb auch wichtig, die Beschreibungen der Daten, also die Metadaten so zu formulieren und gestalten, dass sie die Inhalte nicht nur breiter zugänglich machen, sondern auch auf bereits vorhandene barrierefreie Inhalte verweisen. Aber auch die Daten selbst, sämtliche Guidelines und Nutzungsbedingungen müssen entsprechend gestaltet sein. 
Das Abspeichern von Inhalten in digitalen Langzeitarchivierungssystemen ist oft ein komplexer Vorgang, der möglicherweise sehbehinderten oder blinden WissenschafterInnen ohne Assistenz nicht möglich ist. Diese Einschränkung widerspricht jedoch dem Open Access- bzw. Open Science-Gedanken, der davon ausgeht, dass nicht nur auf sämtliche Daten jederzeit zugegriffen werden kann, sondern dass auch der eigene wissenschaftliche Output, wann immer es möglich ist, auch mit anderen geteilt werden soll. Wenn dies einer Personengruppe verwehrt ist, schließt man sie aus einem wesentlichen Bereich des Wissenschaftsbetriebes aus.

Es ist auffallend, dass nur wenige Personen mit Handicap ein Studium abschließen, verwundert jedoch nicht, wenn man erfährt, wie schwierig es vor allem früher für Menschen mit Beeinträchtigungen war, die Matura abzulegen und überhaupt zum Studium zugelassen zu werden. Mag. ${ }^{a}$ Barbara Levc, erst kürzlich mit dem Dr. Elisabeth Wundsam-HartigPreis ausgezeichnet, hat alle Hürden erfolgreich überwunden und leitet heute das Zentrum Integriert Studieren in Graz. In jungen Jahren erblindet, hat sie das Studium an der Universität Graz abgeschlossen und betreut jetzt gemeinsam mit ihrem Team chronisch kranke oder körperlich beeinträchtigte Studierende. In einem Interview erzählt sie mehr von ihrer Tätigkeit. ${ }^{3}$

Blumesberger: Liebe Frau Mag. . Levc, würden Sie sich bitte vorstellen und von Ihrem Werdegang berichten?

Levc: Ich gehöre noch einer Generation an, die noch nicht mit schulischer Integration aufgewachsen ist, habe in einer Spezialschule für blinde und sehbehinderte Schülerinnen und Schüler die Pflichtschule absolviert und dann noch einen so genannten typischen Blindenberuf im Bereich Telefonie und Schreibtätigkeiten erlernt. Danach habe ich einige Jahre in diesem Beruf gearbeitet und parallel dazu in einem Abendgymnasium maturiert. Es war damals der Anfang der schulischen Integration, alles sehr improvisiert, aber mit viel Pioniergeist und Ausprobieren. Ich war im Gymnasium die erste, die das probiert hat. Da hängt dann ganz viel, vielleicht der ganze Lebensweg, von einer Person ab. Meine erste schüchterne Anfrage bei der Direktorin des Abendgymnasiums wurde von ihr positiv aufgenommen. Sie meinte, das sei ein interessantes Experiment. Das war wirklich lebensentscheidend. Wenn sie das abgelehnt hätte, ich wüsste nicht, ob ich einen weiteren Versuch unternommen hätte. Ich habe anschließend ab 1989 in Graz Erziehungswissenschaften, also im Diplomstudium Pädagogik studiert und mich schwerpunktmäßig auf Sozialpädagogik verlegt. Es 
wurde dann schon während meiner Studienzeit ein Referat für Menschen mit Behinderungen bei der ÖH gegründet, da haben sich Vernetzungen ergeben und aus dem heraus ist dann die Idee einer fixen Stelle, einer Servicestelle für Studierende mit Behinderungen, entstanden. Das war damals eine österreichweite Sache, die Universitäten waren noch nachgeordnete Dienststellen, das ging noch über das Ministerium. Als Resultat sind in weiterer Folge die ersten so genannten Behindertenbeauftragtenstellen eingerichtet worden und für die in Graz habe ich mich beworben und sie erfreulicherweise auch bekommen. Ich bin nun bereits seit 1994 dort und habe gerade das 25jährige Jubiläum hinter mir. Damals war ich noch gar nicht mit dem Studium fertig, denn zunächst kam der Berufseinstieg, dann der Aufbau des Bereichs. Dazwischen habe ich noch geheiratet und einen Sohn bekommen, also da war einiges los in meinem Leben. Ich habe das Studium erst 2005 abgeschlossen und bin derzeit so genannte Behindertenbeauftragte. Der Bereich ist inzwischen gewachsen, es sind viele Studierende dazugekommen, mit 2004 sind wir eine eigene Organisationseinheit geworden, das Zentrum Integriert Studieren. Schön langsam sind immer wieder Mitarbeiterlnnen dazugekommen, ich habe die Leitung und wir sind jetzt mittlerweile fünf Kolleginnen und Kollegen, wobei nur eine davon 40 Stunden da ist, alle anderen sind teilzeitbeschäftigt. Wir sind ein recht flexibles Häufchen hier. Wir haben den Bereich Service für Studierende mit Behinderungen, also auch den Bereich Barrierefreiheit und auch barrierefreie Studienliteratur aufgebaut. Das war sozusagen von Anfang an eines unserer Hauptthemen. Das kam auch daher, dass die UB schon bevor ich als Behindertenbeauftragte angefangen habe, eine Mitarbeiterin hatte, die Studienmaterial gescannt und adaptiert hat. Das war ein Projekt von der damaligen UB-Direktorin, die daran interessiert war und seit 1993 einen Praktikumsplatz hatte. Die Stelle an der UB gibt es immer noch und mit der arbeiten wir ganz intensiv zusammen. Es ist natürlich für eine Person nicht mehr zu stemmen, die gesamte benötigte Literatur zu adaptieren; ein Teil läuft dann auch über uns. Wir arbeiten auch recht viel mit studentischen MitarbeiterInnen zusammen, die scannen und das Korrekturlesen übernehmen. Bei mir hat es sich dann ab ca. 2006 so ergeben, dass ich immer mehr in die Lehre hineingekommen bin. Seit 2009 habe ich eine eigene Vorlesung und werde immer wieder als Gastreferentin eingeladen. Seit 2012 hat sich eine Tätigkeit an der Pädagogischen Hochschule in der Steiermark ergeben, mit einer 25\%igen Anstellung, somit komme ich über die Lehre auch ein wenig in die Forschung hinein.

Blumesberger: Was unterrichten Sie dort? 
Levc: Hauptsächlich Inklusive Pädagogik, also ehemalige Sonderschule, das gibt es ja in dieser Form nicht mehr, sondern man kann zum Volksschullehramt oder zum NMS-Lehramt einen Schwerpunkt Inklusion mit Fokus Behinderung dazu nehmen und dann habe ich noch eine Grundlagenlehrveranstaltung über Diversität und Inklusion. Das müssen alle Lehramtsstudierenden machen, da gibt es viele Gruppen und ich habe zwei davon. Die pädagogischen Hochschulen und Universitäten müssen hier zusammenarbeiten, da gibt es den Verbund Süd-Ost, Steiermark, Kärnten und Burgenland, die haben ihr eigenes Curriculum. Wien ist ein eigener Verbund. Die meisten Dinge sind ziemlich gleich, die Studien sind jedoch unterschiedlich.

Blumesberger: Wie war das, als sie selbst studiert haben, was waren da die größten Hindernisse?

Levc: Es war damals relativ unkompliziert, weil ich mir mit den Professoren eigene Prüfungsvarianten vereinbart habe. Nachdem ich die erste und einzige war, waren die recht aufgeschlossen und wir haben die unterschiedlichsten Möglichkeiten probiert. Zum Teil habe ich schriftliche Prüfungen mündlich gemacht, teilweise habe ich sie schriftlich in einem anderen Raum zu einer anderen Zeit gemacht. Nachdem die Technik noch nicht so entwickelt war, bin ich immer mit einem alten Braille-Notizgerät unterwegs gewesen, aber das konnte man noch nicht mit einem normalen Drucker verbinden, das heißt, ich habe dann noch die kleine Kofferschreibmaschine im Gepäck gehabt und habe das dann übertragen. Ich habe auch einmal im Sekretariat von einem Professor am Computer von der Sekretärin - ohne zu wissen, was ich da schreibe - blind hineingetippt, also es gab die lustigsten Varianten. Im Großen und Ganzen war es improvisierbar. Was zum Teil schwierig war, war komischerweise, dass es manchen Professoren schwer klar zu machen war, dass ich Bücher für Seminare früher haben musste, damit ich sie für mich lesbar machen lassen konnte. Damals war es so, dass es am Beginn eines Seminars einen Büchertisch gab. Dann mussten sich alle ein Thema aussuchen, dabei gab es einen großen Ansturm. Bis ich dann zu einem Buch gekommen bin, waren die besten meistens schon weg. Das war den Lehrenden schwer zu erklären, dass ich mir mit ihnen gerne vorher ein Thema ausgemacht hätte. Damals, am Anfang meiner Studienzeit, habe ich die Bücher zu einem wissenschaftlichen Vorleseservice nach Deutschland geschickt. Die haben das unter wissenschaftlichen Kriterien auf ein Tonband gelesen. Da hat es spezielle Pieptöne gegeben, die man beim Vor- und Rückspulen gehört hat und mit 
denen man dann von einer Seite auf die andere springen konnte. Das hat natürlich Zeit gebraucht. Dann hat es Lehrveranstaltungen gegeben, bei denen ein Studienkollege sehr viel erklärt hat, was man in der Präsentation gesehen hat, vor allem in Statistik. Bei den meisten Lehrveranstaltungen war ohnehin alles klar. Damals, ich habe ab 1989 studiert, gab es kaum großartige Präsentationen. Die meisten haben mit Overheadfolien gearbeitet, da ist man teilweise ohne Sehen ausgekommen. Nur in Statistik war das nicht möglich. In diesem Fall ist dann der Studienkollege mit mir den Stoff durchgegangen. Danach habe ich meine Mitschrift auf den richtigen Stand gebracht. Er hat mir erklärt, wie welche Tabellen aufgebaut sind usw. Es war viel Improvisation, aber es hat funktioniert.

\section{Blumesberger: Das klingt für mich fast einfacher als heute!}

Levc: Es war insofern einfacher, weil das Studium flexibler war. Das Diplomstudium war recht flexibel, es gab Pflichtlehrveranstaltungen, aber man hatte mehr Möglichkeiten sich Dinge auszusuchen, und die Methoden waren noch nicht so komplex und die Schnelllebigkeit von heute gab es noch nicht. Es gab damals Standardbücher, die alle verwendet haben. Jetzt ist alles viel breiter gestreut, vor allem im Zeitschriftenwesen ist alles explodiert. Alles was früher in Sammelbänden publiziert wurde, wird heute in Zeitschriften abgedruckt. Es ist einfach komplexer geworden, die Bachelorstudien sind heute wesentlich verschulter. Ich habe als Zweitfach eine selbstgewählte Fächerkombination gehabt, da habe ich mir hauptsächlich Lehrveranstaltungen aus der Psychologie und Philosophie ausgesucht, deshalb hatte ich damals mehr Möglichkeiten. Aber ich bin natürlich auch manchmal gescheitert. Ich kann mich erinnern, dass ich ein ganzes Semester in einem Psychologieseminar gesessen bin. Zwei Wochen vor dem Prüfungstermin haben alle gejammert, weil es keine Skripten gab. Dann ist der Professor mit einem zusammenkopierten Skriptum gekommen und hat mir keinen späteren Prüfungstermin gegeben. Damit bin ich in diesem Seminar umsonst drinnen gesessen. Wenn das heute jemanden passiert, sage ich: Moment, so geht es nicht! Aber als junge Studentin, ohne Unterstützung im Hintergrund, wusste ich nicht, wie ich mich da hätte wehren sollen. Es hat auch immer wieder Lehrveranstaltungen gegeben, bei denen ich aufgehört habe, weil ich bemerkt habe, es geht einfach nicht. Im Großen und Ganzen ist es aber eigentlich recht gut gelaufen.

Blumesberger: Mich macht es immer betroffen, wenn ich höre, dass Sie die einzige waren, wo waren die anderen? 
Levc: Ja, das war 1989, da hat es noch keine Möglichkeit gegeben, auf dem regulären Weg die Matura zu machen. Man musste nach einer Pflichtschulausbildung zu Maturaschulen oder Abendschulen gehen.

Zwei Jahre nach mir hat ein sehbehinderter, junger Mann mit dem Studium begonnen. Er war einer der ersten, die regulär maturiert haben. In der Steiermark ist der Beginn der schulischen Integration für Kinder mit Sehbehinderung ca. mit 1985 angesetzt, für blinde Kinder mit ca. 1989. Schön langsam hat es sich dann entwickelt.

Blumesberger: Aber es gibt immer noch zu wenige!

Levc: Ja, es gibt zu wenige. Die Rahmenbedingungen mit Integration und Begleitlehrer sind nicht immer so gut, dass es in den Schulen reibungslos funktioniert, nämlich, dass sie abgesehen vom Lernen auch einen vernünftigen Draht zu ihren Schulkollegen haben. Schwierig ist es immer noch, aber schon um einiges besser.

Blumesberger: Kommen wir zum Thema Open Science, Open Data. Wir sprechen so oft von offener Wissenschaft, von frei verfügbaren Daten. Was aber kaum ausgesprochen wird, ist die Tatsache, dass die Daten für alle, auch für Menschen mit Behinderungen offen sein müssen. Deshalb wollte ich Sie fragen, wie Sie zum Beispiel mit der Vorbereitung für Lehrveranstaltungen umgehen? Wie kommen Sie zu Informationen?

Levc: Ich hantle mich oft von einer Information zur nächsten. Ich habe natürlich den Luxus, dass ich hier an der Uni meine eigenen Ressourcen nutzen kann. Wenn ich einen Zeitschriftenartikel benötige, kann ich den Kollegen in der Bibliothek bitten, dass er mir den Artikel recherchiert und wenn nötig auch gleich adaptiert. Ich suche mir aber auch über das Web Informationen zusammen, habe mir auch schon einige Bücher als E-book bestellt, das ist dann so ein Mix. Die Recherche ist manchmal recht kompliziert. Ich gehöre auch zu den Menschen, die bevor sie einen Text lesen, das Literaturverzeichnis lesen, dann weiß ich schon vorher was mich noch interessieren würde.

Blumesberger: Ich kann mir gut vorstellen, dass Sie durch Ihre Netzwerke eher Unterstützung finden, aber das trifft für externe Lehrende nicht zu. Ich kenne zum Beispiel keine Personen mit Behinderungen, die unterrichten. Da fragt man sich dann schon, wie das sein kann. Warum gibt es die nicht? 
Levc: Das kommt jetzt erst langsam ins Bewusstsein. Mit Studierenden mit Behinderungen hat man sich bereits gedanklich angefreundet, aber dass nach dem Studium das Leben auch noch weitergeht und dass es viele Personen gibt, die eine wissenschaftliche Karriere anstreben würden und auch das Zeug dazu hätten, das kommt erst so langsam. Es werden jetzt gerade im Rahmen des Projekts PromoLi (Promotion ohne Limit) ${ }^{4}$ Doktoratsstellen vergeben, an insgesamt 8 Universitäten, bezahlt wird das von der Uni und vom Sozialministerium. Damit sollen mehr Personen mit Beeinträchtigungen in den Betrieb hineinwachsen. Das wird sicher für die Unis ein wichtiger Lernprozess, was man denn außer einem Schreibtisch und einem barrierefreien Hörsaal noch so alles braucht.

Blumesberger: Das wird aber sicher noch eine Zeit dauern...

Levc: Was man noch dazu sagen muss, ist, dass die digitalen Ressourcen, nicht nur für blinde und sehbehinderte Personen enorm hilfreich sind, sondern auch für Leute mit Körperbehinderungen, die nicht mit Büchern hantieren können. Wir haben einige normal sehende Studierende, die ihre Literatur am Tablet haben, um mit Wischbewegungen blättern zu können, die Handmobilität würde für gedruckte Bücher nicht ausreichen. Auch Leute mit starker Lese-Rechtschreibschwäche profitieren davon. Sobald man etwas als Datei hat, kann man es auch in ein Audiodokument umwandeln.

Blumesberger: Sie haben Recht. Barrierefreiheit ist nicht nur für eine kleine Gruppe interessant, sondern für uns alle. Arbeiten Sie auch mit Daten? Sind Sie schon mit Repositorien in Berührung gekommen?

Levc: Nein, nicht wirklich. Ich arbeite vor allem mit Literatur. Man hat dann halt seine gut ausgetretenen Wege, auf denen man recherchiert und weiß vielleicht gar nicht, was es sonst noch so alles gibt. Ich habe jetzt als Gastvortragende eine Kollegin von der Bibliothek kennengelernt und war ganz begeistert, welche weiteren Recherchemöglichkeiten es gibt.

Blumesberger: Sie unterstützen ja an der Universität Studierende mit Beeinträchtigungen. Wie kann man sich das vorstellen?

Levc: Es gibt mal das Erstgespräch. Während des Studiums gibt es einen eingespielten Ablauf, wenn Leute Bedarf an einer barrierefreien Literatur 
haben. Nachdem es ziemlich viele sind, haben wir uns über SharePoint ein Formular eingerichtet, wo die Leute ihren Bücherbedarf eintragen. Wenn das in der Bibliothek verfügbar ist, leiht es der Bibliothekskollege aus und scannt es eventuell gleich. Danach wird es zum Korrekturlesen an studentische Mitarbeiterlnnen vergeben, eine Kollegin koordiniert den Prozess, der schon ziemlich standardisiert ist. Die Bücher, die wir bereits adaptiert haben, sind in einer Datenbank gespeichert. Die Studierenden können recherchieren, ob es das Buch schon gibt, dann erhalten sie nach Vorlage eines Behindertenausweises und der Unterschrift mit dem Einverständnis, die Datei nicht an Dritte weiterzugeben, das adaptierte Dokument. Es gibt aber auch kurzfristige Hilfen, vor allem für Studierende der Sprachen oder Dolmetsch, die für eine Übung adaptierte Texte benötigen. Da gibt es die Funktion „Zettel hochladen“ im SharePoint. Die Studierenden erhalten oft schon von den Vortragenden die Datei oder die Präsentationen für die nächste Lehrveranstaltung, die man dann nur noch barrierefrei machen muss. Ich bemühe mich inzwischen sehr, das Wissen über Barrierefreiheit zu verbreiten, denn je besser die Aufbereitung in dieser Hinsicht schon ist, desto rascher können die Dateien barrierefrei gemacht werden. Bei einer Powerpointpräsentation wird man immer etwas nachbearbeiten müssen, aber ein PDF kann man schon recht einfach barrierefrei erstellen.

Blumesberger: Was würden Sie sich für die Zukunft wünschen, wo sehen Sie einen Bedarf?

Levc: Es gibt Standards für Barrierefreiheit im Netz und für barrierefreie Dokumente. Ich würde mir wünschen, bevor irgendetwas in irgendeiner Form online gestellt werden darf, muss es durch einen Filter, der die Barrierefreiheit prüft. Damit wären wir alle Probleme los, auf einen Schlag! Öffentliche Angebote sollten eigentlich ohnehin schon barrierefrei sein.

Blumesberger: Was würden Sie jungen Menschen mit Behinderungen heute bzgl. Berufraten?

Levc: Man kann das schwer generalisieren, aber man sollte immer auf die eigenen Interessen hören und nicht auf das Umfeld. Beim Lehramtsstudium, das ja lange Zeit für Personen mit Behinderungen nicht möglich war, weil die Voraussetzung der körperlichen Eignung im Wege gestanden ist. Das ist mit dem Behindertengleichstellungsgesetz gefallen. Oft wird jungen Leuten abgeraten, den Lehrerlnnenberuf anzustreben, weil nicht Betroffene sich nicht vorstellen können, wie es möglich ist, zum Beispiel auf 
eine ganze Klasse aufzupassen. Dabei wird die Kreativität dieser Menschen nicht beachtet. Eine gute Lehrpersönlichkeit muss auch ohne Behinderungen nicht den Schülerlnnen nachrennen. Das ist aber ein Gebiet, das sich erst langsam entwickelt und ausgebaut werden muss. Man sollte den eigenen Interessen folgen. Es mangelt auf der anderen Seite aber auch an Berufsmöglichkeiten im mittleren Berufsspektrum. Es gibt immer wieder Studierende, bei denen wir den Eindruck haben, dass sie aus Mangel an anderen Perspektiven an der Uni sind, sich aber durchquälen. Sie wären vielleicht besser in einer Lehrstelle aufgehoben. Da ist dann immer die Frage, inwieweit sie im Berufsleben, mit dem was sie studiert haben, ankommen werden.

Blumesberger: Liebe Frau Mag. . Levc, vielen herzlichen Dank für das Gespräch!

\section{Fazit}

Das Gespräch mit Barbara Levc zeigt, dass es bzgl. offener Wissenschaft und offener Daten noch einiges zu tun gibt. Es ist noch ein weiter Weg bis Barrierefreiheit auf allen Ebenen als selbstverständlich angesehen und mitgedacht wird und wir wirklich von fairen FAIR-Data sprechen können. Zugleich müssen aber auch die Bildungsmöglichkeiten so gestaltet werden, dass es selbstverständlich ist, dass Schülerlnnen mit körperlichen Beeinträchtigungen die Universität offensteht, dass nicht nur Studierende sondern auch Lehrende mit Handicaps die Regel und nicht bloß die Ausnahme sind, dass sämtliche technischen Möglichkeiten ausgeschöpft werden, um allen Menschen die gleiche Chancen im Rahmen des Wissenschaftssystems einzuräumen.

Mag. ${ }^{a}$ Dr. ${ }^{\text {in }}$ Susanne Blumesberger

ORCID: https://orcid.org/0000-0001-9018-623X Universität Wien, Bibliotheks- und Archivwesen E-Mail: susanne.blumesberger@univie.ac.at

Mag. ${ }^{a}$ Barbara Levc Universität Graz, Zentrum Integriert Studieren E-Mail: barbara.levc@uni-graz.at 
1 Universität Graz, Zentrum Integriert Studieren: https://integriert-studieren.uni-graz.at/

2 Siehe die Forschungsdatenpolicy der Technischen Universität Wien: https://www.tuwien.at/fileadmin/Assets/forschung/Zentrum_Forschungsdatenmanagement/Policy_Forschungsdatenmanagement.pdf

3 Das Interview fand am 29.4.2019 per Telefon statt.

4 https://uniko.ac.at/projekte/promoli/ 